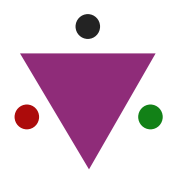

IJCRR

Section: Healthcare

Sci. Journal Impact

Factor: 6.1 (2018)

ICV: 90.90 (2018)

\section{cc) (i) (3)}

Copyright@IJCRR

\title{
Accuracy of Intra-Operative Frozen Section Consultation of Gastrointestinal Biopsy Samples in Correlation with the Final Histopathological Diagnosis
}

\author{
Mohita Ray ${ }^{1}$, Pranita Mohanty ${ }^{2}$, Sunil Agarwal ${ }^{3}$, Debahuti Mohapatra ${ }^{4}$
}

'Senior Resident, Department of Pathology, IMS \& SUM hospital, Siksha "O” Anusandhan University (Deemed to be), K8, Kalinga Nagar, Bhubaneswar-751003, Odisha, India; ${ }^{2}$ Professor, Department of Pathology, IMS \& SUM Hospital, Siksha “O” Anusandhan University (Deemed to be), K8, Kalinga Nagar, Bhubaneswar-751003, Odisha, India; 3Professor, Department of Surgical Oncology, IMS \& SUM Hospital, Siksha "O" Anusandhan University (Deemed to be), K8, Kalinga nagar, Bhubaneswar-751003, Odisha, India; “Professor \& HOD, Department of Pathology, IMS \& SUM Hospital Siksha "O” Anusandhan University (Deemed to be), K8, Kalinga Nagar, Bhubaneswar-751003, Odisha, India.

\section{ABSTRACT}

Introduction: Frozen section (FS) is an intraoperative diagnostic tool which provides the operating surgeon with a rapid diagnosis, helping him to make a timely decision which may have a significant impact on patient management \& prognosis. Hence the efficiency of this technique has to be periodically reviewed to ensure that the quality of the sections and agreement between frozen section diagnosis and final diagnosis remain at an acceptable level.

Objective: To assess accuracy, sensitivity \& specificity of Intra-operative frozen section in the diagnosis of a lesion \& in determining the adequacy of surgical resection margins in gastrointestinal surgeries.

Methods: Total 220 cases and 521 intra-operative FS specimen during gastro-intestinal surgeries were analysed. The samples were sent for intraoperative diagnosis and margin assessment. The initial FS report was then correlated with the final histopathology report and the concordance rate \& reasons for discrepancies were analysed.

Results: After the correlation of all the specimen with final histopathology, the diagnosis was conclusive in $99.2 \%$ (215 cases) \& deferred in $0.8 \%$ (5 cases). The cases with conclusive diagnoses showed a concordance rate of $91.1 \%$ for all cases and $95.9 \%$ for all specimens (excluding deferrals). Sensitivity and specificity of FS diagnosis as compared to histopathology was found to be $89.3 \%$ and $98.4 \%$ respectively.

Conclusion: The accuracy, sensitivity, specificity of frozen section diagnosis in this study are comparable with most international quality control statistics for frozen sections. Keeping the limitations and pitfalls in mind, FS technique is very reliable in good hands.

Key Words: Frozen section, Intra-operative consultation, Concordance, Histopathology

\section{INTRODUCTION}

Tissue obtained after the operation is usually assessed by histopathologists on the following day after adequate formalin fixation of the specimen. However, during certain circumstances, the surgeons require the urgent pathologic information while the patient is still on the operative table, to take an intra-operative or peri-operative decision on the treatment. Hence, they request an intra-operative consultation on a tissue that arouses doubt. FS analysis as an intra-operative consultation is a powerful tool that can help guide surgical decision making by providing quick diagnosis, tissue recognition and extent of resection necessary. Thus it helps in the making of therapeutic decisions, thereby sparing the patient from possible re-operation and reducing the length and cost of hospitalization. ${ }^{1,2}$

Further, the decision to perform a radical surgery of lymph nodes is entirely dependent on the frozen section. Frozen section help in rapid diagnosis of the lesion and intra-operative histological evaluation of tumour resection borders. This is done without prolonging the surgery and thus acts as a

\section{Corresponding Author:}

Dr. Mohita Ray, Senior Resident, Department of Pathology, IMS and SUM hospital, Siksha "O" Anusandhan University (Deemed to be), K8, Kalinga nagar, Bhubaneswar-751003, Odisha, India; Mobile: 9438664462; 6370200839; Email: mohitaray@soa.ac.in

ISSN: 2231-2196 (Print) ISSN: 0975-5241 (Online)

Received: $15.07 .2020 \quad$ Revised: 23.09 .2020

Accepted: 14.11 .2020

Published: 05.01 .2021 
guide to the surgeon in making a therapeutic decision, while the patient is still on the operating table..$^{3-5}$

In cases where tumour-free margins have been achieved at the time of surgery, lower local tumour recurrence rates and improved survival have been reported. ${ }^{6-10}$ Intra-operative frozen section analysis of margins is widely employed to assist in complete tumour extirpation. Frozen section diagnosis of surgically resected tumours and tissues has become a regular practice over the past 60 years. ${ }^{11-13}$ Hence it is very essential to evaluate the quality \& accuracy of FS reporting.

\section{MATERIALS AND METHODS}

The current study was carried out in the department of cytology \& histopathology of IMS \& SUM Hospital, Bhubaneswar over 3 years from 2014 to 2017 . A prospective analysis of all the intraoperative frozen sections performed during gastrointestinal surgeries was done \& a total of 220 cases very studied which yielded 521 specimens. Tissue was sent from the operation theatre in normal saline to the central cytology laboratory, where the sample-receiving time \& gross findings were noted, and sections were taken. Blocks were cut on LEICA CM1860 cryostat. The slides were stained with haematoxylin and eosin (H \& E) stain and studied. In cases of positive margins, results were informed to surgeon over the phone and revised margins were sent which were subsequently studied \& yielded final FS report. The final results were informed to the operation theatre over the phone. Frozen tissue as well as any remaining non-frozen tissue were then fixed in $10 \%$ formalin solution \& sent to histopathology lab to make paraffin embed sections \& H\&E stained slides.

The FS report given at the time of surgery was compared to the final histopathology report of the permanent sections $\&$ the accuracy rate, sensitivity and specificity of the frozen section reporting were determined in comparison to the routine histopathology reporting. Any discrepancy was noted and all cases which were discordant as regards the benign versus malignant, and negative versus positive margins were reviewed. The review process included the re-examination of all slides with the categorization of the reasons for discordance in three categories, i.e., Interpretative errors, sampling errors \& technical errors. Patients of both sex, of age group 5-85 years undergoing gastro-intestinal surgery with informed consent were included in the study. Other sampling techniques like endoscopic biopsy, fine needle aspiration, brushings, lavage, peritoneal, pancreatic or bile fluid analysis were excluded.

The study design was approved by the Research Ethics Committee of IMS \& SUM Hospital, Bhubaneswar. Any discrepancy was noted and all cases which were discordant as regards the benign versus malignant, and negative versus positive margins were reviewed.

\section{RESULTS}

Age distribution of cases showed that the maximum number of cases operated was around 60 years of age (mode $=$ 60 years). Clustering of cases was seen from 35 years of age to 70 years of age. Age of the youngest patient was 11 years old female, with clinical diagnosis Mucoepidermoid Carcinoma, whose $3^{\text {rd }}$ Dimensional margin was submitted for intraoperative frozen section, which was confirmed to be free of tumour. Age of the eldest patient was 84 years male, with a clinical diagnosis of Carcinoma left buccal mucosa, whose intraoperative frozen section biopsy was submitted to establish primary malignancy, \& was subsequently diagnosed to have Pseudoepitheliomatous hyperplasia. FS was done mostly in male patients $(70 \%)$. A maximum number of Gastrointestinal cases sent for Intraoperative frozen section consultation were sent with a clinical diagnosis of the malignant lesion $(97.27 \%)$. In the indication -wise distribution of the 220 cases, 111 cases (111 specimens) were sent to establish/ confirm a pathology/ primary malignancy, 90 cases (372 specimens) to determine the adequacy of resection margins \& 19 cases (38 specimens) to establish/confirm metastatic malignancy. Hence, most of the cases were sent with the indication to establish/confirm a pathology/primary malignancy $(50 \%)$.

There were total 71 cases diagnosed as malignant on final paraffin embed sections (including diagnoses of suspicious of malignancy) which comprised of $66.98 \%$ of the final diagnoses of the cases sent with an indication to establish/ confirm primary pathology/malignancy. Squamous cell carcinoma (oral cavity) was the most commonly diagnosed malignant lesion (33 cases, 31.1\%). Most of the specimen for Intraoperative FS were submitted for surgeries of the Oral cavity $(51.14 \%)$ followed by Stomach $(17.80 \%)$, large intestine $(6.78 \%)$, gall bladder(5.23\%) \& periampullary region(3.87\%) (Figure.1).

Overall \% Concordance/ Accuracy of FS in Gastrointestinal surgeries was $95.9 \%$. The sensitivity and specificity of FS were found to be $89.3 \%$ (95\% CI: $82.9,93.9)$ and $98.4 \%$ (95\% CI: 96.6, 99.4) respectively. Receiver operating curve (ROC) area was found to be 0.94 (95\% CI: $0.67,0.75)$ thus justifying that FS can be used as a diagnostic test against the gold standard for intra-operative samples collected during surgeries of the Gastro-intestinal tract. The positive predictive value and negative predictive value for FS in detecting any pathologies were found to be $95.4 \%$ (95\% CI: $90.3,98.3$ ) and $96.1 \%(93.7,97.8)$ respectively. The positive likelihood ratio (LR) of FS was found to be very strong and it was 56 (95\% CI: $25.2,124)$. The test has very strong likelihood ratio $(>10)$ which means that sections which are positive with Histopathology are 81 times more likely to have positive result also with FS as compared to the sections with negative Histopathology results. The negative likelihood ratio of FS 
was found to be 0.109 (95\% CI: 0.07, 0.18). The test result was strong (near to 0.1 ) which means that specimen found to be negative with Histopathology are 0.1 times likely to have a negative result also with FS as compared to the positive results with Histopathology. (Table. no. 1)

In the 106 cases submitted with an indication to Establish/ confirm Primary pathology/malignancy (excluding deferred cases), the FS diagnoses showed high concordance (84.9 $\%)$ with the final Permanent section diagnoses. Figure No. 2, 3, 4 show images of FS \& subsequent histopathology of the specimen with concordant diagnoses. In the 372 margins submitted for evaluation, the overall accuracy of FS was $99.7 \%$ with a sensitivity of $95.24 \%$ \& specificity of $100 \%$. The overall accuracy of FS in establishing/ confirming metastatic malignancy was $93.3 \%$ with a sensitivity of $100 \%$ \& specificity of $80 \%$. Figure No.5 shows a colonic serosal nodule diagnosed as metastatic adenocarcinomatous deposits on FS, which was confirmed on histopathology. ${ }^{23-25}$

There were 21 discordant diagnoses (19 cases) which comprised of $4.1 \%$ of all the conclusive frozen section diagnoses. Of these 19 discordant diagnoses, false-positive were 13 $\&$ false negative were 6 . Of all discordant diagnoses, 10 were due to misinterpretation $(52.63 \%) \& 9$ were due to sampling error $(47.37 \%)$.

\section{DISCUSSION}

Majority of the cases where FS specimen were sent was for confirmation of diagnosis (around 50\%), followed by margin assessment and lymph node metastasis (rest 50\%) which is in line with other studies. ${ }^{11-14}$ Since the majority of the resection cases were neoplastic, there was the need to ensure that entire pathology has been removed. Complete awareness of the indications for FS should be there in the operating surgeon as well as pathologists to ensure that appropriate requests are attended to. Only then errors can be reduced. . $^{15,16,17}$ Oral cancer was among the most common form of GI cancers encountered, which is in line with epidemiology. (figure. 1)

Our study showed a concordance rate of $91.1 \%$ for all cases and $95.9 \%$ for all specimens (excluding deferrals). This was found to be in line with other studies. (Table 2) The concordance rate has always been more than $90 \%$, with a usual range of $94 \%$ to $97 \%$. Mishra S et al conducted the study comparing the accuracy of FS with Histopathology and found a concordance rate of $96.2 \%$. ${ }^{18,19}$ Studies conducted by Chbani et al., had an accuracy rate of $95 \%$, while that of Geramizadeh B et al. had an accuracy of $96.7 \% .{ }^{15,20}$ If the accuracy of GIT specimens is considered, it varied depending on the number of samples. Geramizadeh B 'et al' had an accuracy of only $75 \%$ for GIT specimens, as there were only 4 samples (Table 2)..$^{20,21}$
Sensitivity and specificity of FS diagnoses as compared to Histopathology was found to be $89.3 \%$ and $98.4 \%$ respectively. Thus, FS specimens were found to be highly specific and can limit the number of false negatives to a minimum. The study conducted by Patil P et al., had higher sensitivity of $97 \%$ as compared to our study while the specificity is almost the same (98.4\%). ${ }^{12}$ However, the study does not mention 95\% CI levels, which could have probably overlapped with that of ours (95\% CI for sensitivity for our study: 82.9 , 93.9).

In our study, the overall accuracy of FS in margin assessment was very high of $99.7 \%$ with a sensitivity of $95.24 \%$ \& specificity of $100 \%$ which was comparable to the findings of DiNardo 'et al', who analysed FS margins in head and neck cancer surgeries \& reported an accuracy, sensitivity \& specificity of $98.3 \%, 88.8 \% \& 98.9 \%$ respectively. ${ }^{22,26}$

\section{CONCLUSION}

- The accuracy, sensitivity and specificity of FS diagnosis as compared to Histopathology was found to be $95.9 \%, 89.3 \%$ and $98.4 \%$ respectively which are comparable with most international quality control statistics for frozen section

- Receiver operating curve (ROC) area was found to be 0.94 (95\% CI: $0.67,0.75)$ thus justifying that FS can be used as a diagnostic test against histopathology for intra-operative samples collected during surgeries of the Gastro-intestinal tract.

- As compared to other studies, our study could even throw light on likelihood ratios, predicting chances of a slide being positive or negative in FS depending on the results of Histopathology. LR+ and LR- overall was found to be 56 and 0.109 , strongly indicating that FS can be a better diagnostic test as compared to Histopathology if other steps are taken to reduce discordance (through error reduction).

- Keeping the limitations and pitfalls of FS in mind, it should be noted that the FS technique is very reliable in good hands.

\section{ACKNOWLEDGMENT}

The author acknowledges the help received from Professor and Head, Department of for her guidance. I am also thankful to my Guide for her kind co-operation \& meticulous supervision of the work. I would like to give my special thanks to all the technicians of Histopathology \& cytology Section, for helping me while conducting the present study. The author is also grateful to authors / editors / publishers of all those articles, journals and books from where the literature for this article has been reviewed, discussed \& cited.

Conflict of interest: None 


\section{Financial support: Nil}

\section{ABBREVIATIONS}

FS- Frozen section

GIT- Gastrointestinal tract

CI- Confidence interval

LR- Likelihood Ratio

\section{REFERENCES}

1. Howanitz PJ1, Hoffman GG, ZarboRJ.The accuracy of frozensection diagnoses in 34 hospitals. Arch Pathol Lab Med 1990 Apr;114(4):355-9.

2. Kaufman Z, Lew S, Griffel B, Dinbar A. Frozen section diagnosis in surgical pathology- A prospective analysis of 526 frozen sections. Cancer 1986;57(2): 377-379.

3. Bahr W, Stoll P. Intraoperative histological evaluation of tumour resection borders without prolonging surgery. Int J Oral Maxillofac Surg 1992;21(2): 90-91

4. Ravasz LA, Slootweg PJ, Hordijk GJ, Smit F, van der Tweel I. The status of the resection margin as a prognostic factor in the treatment of head and neck carcinoma. J Crani- Maxillofac Surg 1991;19:314-318.

5. Jones AS, Bin Hanafi Z, Nadapalan V, Roland NJ, Kinsella A, Helliwell TR. Do positive resection margins after ablative surgery for head and neck cancer adversely affect prognosis? A study of 352 patients with recurrent carcinoma following radiotherapy treated by salvage surgery. Br J Cancer 1996;74(1):128132.

6. Chen TY, Emrich LJ, Driscoll BA. The clinical significance of pathological findings in surgically resected margins of the primary tumour in head and neck carcinoma. Int $\mathrm{J}$ Radiat Oncol Biol Phys 1987;13:833-837.

7. Cook A, Jones AS, Phillips DE, Soler Lluch E. Complications of tumour in resection margins following surgical treatment of squamous cell carcinoma of the head and neck. Clin Otolaryngol 1993;18:37-41.

8. Looser KG, Shah JP, Strong EW. The significance of "positive" margins in surgically resected epidermoid carcinomas. Head Neck Surg 1978;1:107-111.

9. MacCarty WC, The diagnostic reliability of frozen sections. Am J Pathol 1929;5(4):377-380.5.

10. Peters PM. Frozen section diagnosis. $\mathrm{Br}$ Med J 1959;1(5133):1321-1322.

11. Tangled A, Shrivastava V, Joshi A. Analysis of frozen section in correlation with surgical pathology diagnosis. Int J Res Med Sci 2019;7:2312-7.
12. Patil P, Shukla S, Bhake A, Hiwale K. Accuracy of frozen section analysis in correlation with surgical pathology diagnosis. Int J Res Med Sci 2015;3:399-404.

13. Chatelain D, Schildknecht H, Trouillet N, Brasseur E, Darrac I, Regimbeau JM. Intraoperative consultation in digestive surgery. A consecutive series of 800 frozen sections. J ChirurgieViscérale 2012; 149(2) :146-155.

14. Ahmad Z, Barakzai MA, Idrees R, Bhurgri Y. Correlation of intraoperative frozen section consultation with the final diagnosis at a referral centre in Karachi, Pakistan. Ind. J Pathol Micro 2008;51:469-73

15. Laila Chbani, Sekal Mohamed, Tawfik Harmouch; Quality assessment of intraoperative frozen sections: An analysis of 261 consecutive cases in a resource-limited area: Morocco. Sci Res Health 2012;4: 433-435

16. Valerie A. White; Intraoperative Consultation/Final Diagnosis Correlation Relationship to Tissue Type and Pathologic Process; Arch Pathol Lab Med 2008;132:29-36

17. McIntosh ER. Frozen section: guiding the hands of surgeons? Ann Diagn Pathol 2015; 2(4): 123-6.

18. HasnanJaafar, Intra-Operative Frozen Section Consultation: Concepts, Applications And Limitations, Malay. J Med Sci 2006;13(1): 4-12.

19. Saumya Mishra et al., Qualitative Comparative Study of Frozen Section with Routine Histological Technique; Nat J Lab Med. 2016.

20. Bita Geramizadeh, Taghi Rezai Larijani, Seyed-Mohammad Owji; Accuracy of intra-operative frozen section consultation in the south of Iran during four years; Ind J Path Micro 2010; 53(3): 414-417

21. Sams SB, Wisell JA. Discordance Between Intraoperative Consultation by Frozen Section and Final Diagnosis: A Classification Model to Guide Quality Improvement.; Int J Surg Pathol. 2016; 8: 341-345.

22. Di N. Frozen Section Margins in Head and Neck Cancer Surgery . Laryngoscope. 2000; 110:1773-1776.

23. Khoo JJ1.An audit of intraoperative frozen section in Johor.Med J Malaysia. 2004 Mar;59(1):50-5.

24. Ferreiro JA, Myers JL, Bostwick DG. Accuracy of frozen section diagnosis in surgical pathology: review of a 1-year experience with 24,880 cases at Mayo Clinic Rochester. Mayo Clin Proc 1995;70(12):1137-1141.

25. Khaled A, Agrawal L, Nasir TA; Correlation of Intra-Operative Frozen Section Consultation With the Final Diagnosis At a Tertiary Referral Center in Dhaka. Pulse 2011; 5(2): 22-25.

26. Rafael DenadaiPigozzi Da Silva1; Luís Ricardo MartinhãoSouto, Tcbc-Sp2; Graziela De Macedo Matsushita3; Marcus De Medeiros Matsushita3. Diagnostic accuracy of frozen section tests for surgical diseases. Rev Col Bras Circ 2011; 38(3): 149-154. 
Table 1: Overall Accuracy of FS diagnosis in surgeries of GIT

\begin{tabular}{r|rr|r}
$\begin{array}{r}\text { True } \\
\text { disease } \\
\text { status }\end{array}$ & \multicolumn{2}{|c|}{ Test result } & Total \\
\hline Neg. & Pos. & 6 & 376 \\
Normal & 370 & 125 & 140 \\
Abnormal & 15 & 131 & 516
\end{tabular}

\begin{tabular}{|c|c|c|c|c|}
\hline Prevalence & $\operatorname{Pr}(\mathrm{A})$ & $27 \%$ & $23 \%$ & $31.2 \%$ \\
\hline Sensitivity & $\operatorname{Pr}(+\mid A)$ & $89.3 \%$ & $82.9 \%$ & $93.9 \%$ \\
\hline Specificity & $\operatorname{Pr}(-\mid N)$ & $98.4 \%$ & $96.6 \%$ & $99.4 \%$ \\
\hline ROC area & (Sens. + Spec.) $/ 2$ & .938 & .912 & .965 \\
\hline Likelihood ratio (+) & $\operatorname{Pr}(+\mid A) / \operatorname{Pr}(+\mid N)$ & 56 & 25.2 & 124 \\
\hline Likelihood ratio (-) & $\operatorname{Pr}(-\mid A) / \operatorname{Pr}(-\mid N)$ & .109 & .0675 & .176 \\
\hline Odds ratio & $\operatorname{LR}(+) / \operatorname{LR}(-)$ & 514 & 198 & 1326 \\
\hline Positive predictive value & $\operatorname{Pr}(\mathrm{A} \mid+)$ & $95.4 \%$ & $90.3 \%$ & $98.3 \%$ \\
\hline Negative predictive value & $\operatorname{Pr}(\mathrm{N} \mid-)$ & $96.1 \%$ & $93.7 \%$ & $97.8 \%$ \\
\hline
\end{tabular}

Table 2: Different studies showing the accuracy of Frozen Section as compared to Histopathology

\begin{tabular}{|c|c|c|c|c|c|}
\hline Site of lesion & No. of specimen & $\begin{array}{c}\text { Accuracy/ } \\
\text { Concordance rate }\end{array}$ & Sensitivity & Specificity & Reference, Remarks \\
\hline overall & 1042 & $95.4 \%$ & & & 21 \\
\hline Overall & 83 & $91.5 \%$ & $85.7 \%$ & $97 \cdot 9 \%$ & 11 \\
\hline Overall & 24,880 & $97.8 \%$ & & & 24 \\
\hline Overall & 215 & $97.5 \%$ & $97.9 \%$ & $97.1 \%$ & 23 \\
\hline Head and neck & 420 & $98.3 \%$ & $88.8 \%$ & $98.9 \%$ & 22, Margins \\
\hline Overall & 52 & $96.2 \%$ & & & 19 \\
\hline GI cases & 15 & $93.3 \%$ & & & \\
\hline Overall & 176 & $98.3 \%$ & $98.85 \%$ & & 25 \\
\hline GI cases & 3 & $100 \%$ & & & \\
\hline Overall & 100 & $96.9 \%$ & $97.2 \%$ & $96.3 \%$ & 12 \\
\hline Oral cavity & 23 & $100 \%$ & & & \\
\hline GIT & 5 & $100 \%$ & & & \\
\hline Overall & 433 & $93 \cdot 3 \%$ & & & 26 \\
\hline GIT & 12 & $100 \%$ & & & \\
\hline Overall & 759 & $96.7 \%$ & & & 20 \\
\hline GIT & 4 & $75 \%$ & & & \\
\hline GIT & 800 & $96.6 \%$ & & & 13 \\
\hline Overall & 2811 & $96.9 \%$ & & & 16 \\
\hline GIT & 521 & $95.9 \%$ & $89.3 \%$ & $98.4 \%$ & \\
\hline
\end{tabular}




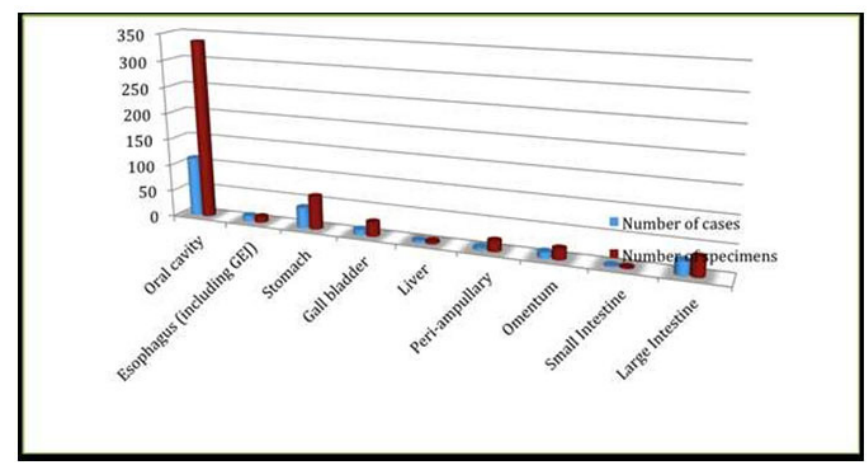

Figure 1: This bar diagram shows that most of the specimen for Intraoperative FS were submitted for surgeries of the Oral cavity $(51.14 \%)$ followed by Stomach $(17.80 \%)$.

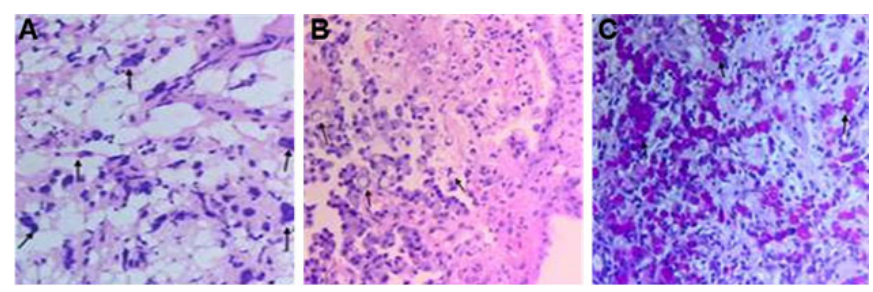

Figure 2: Adenocarcinoma, Stomach (signet ring cell type) A. FS from ulcerated growth in gastric fundus reported as Adenocarcinoma, Stomach. (H\&E, x100). B. Permanent sections also showing features of Adenocarcinoma Stomach (Signet ring cell type) (H\&E, x400). C. PAS +ve signet ring cells. (PAS, x400).
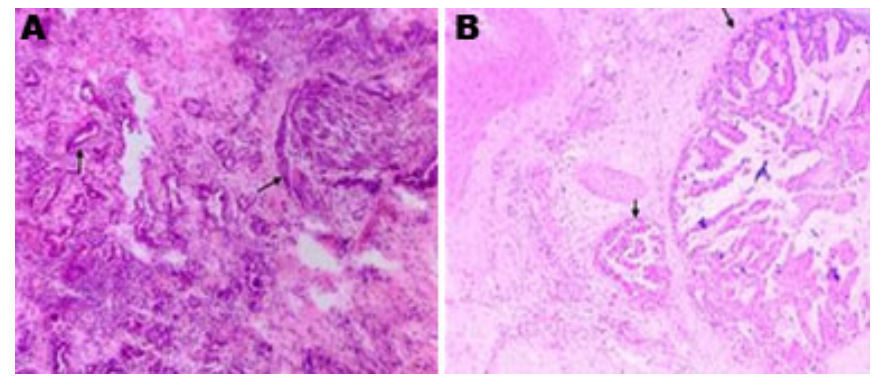

Figure 3: Adenocarcinoma Gall bladder. A. FS from Gall bladder showing features of Adenocarcinoma, Gall Bladder. (H\&E, $x 100)$. B. Deeper sections from the corresponding permanent section showing Infiltrating Adenocarcinoma, Gall Bladder. (H\&E, x100).
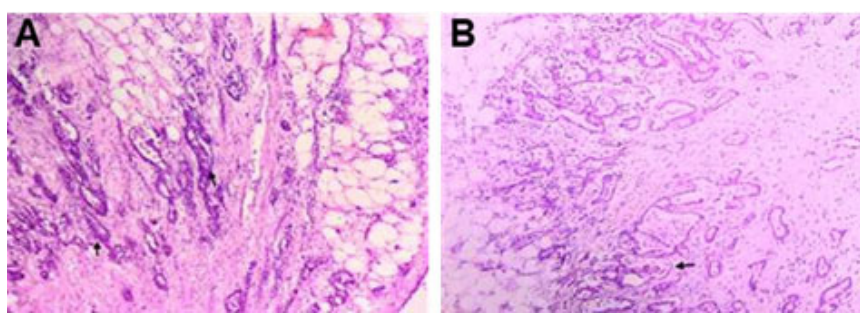

Figure 4: Tubular Adenoma, colon. A. FS from a case of Recurrent Carcinoma Colon with multiple polyp. showing features of Tubular Adenoma with High grade Dysplasia. (H\&E, $x 100) B$. Subsequent permanent sections from the same case showing features of Tubular Adenoma with High grade Dysplasia. (H\&E, x100).
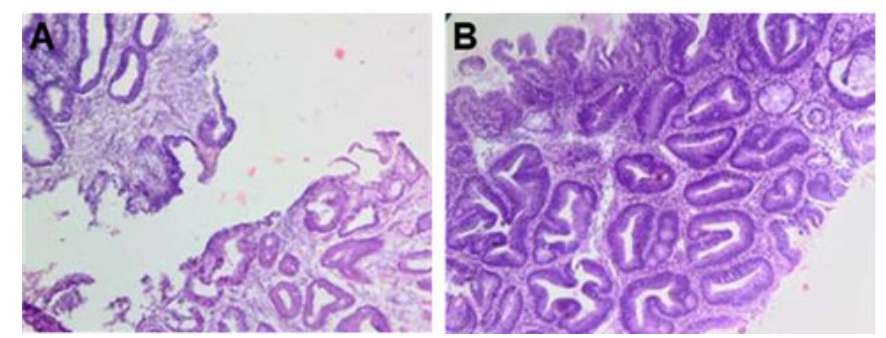

Figure 5: Metastatic serosal nodule. A. FS from colonic serosal nodule showing features of Metastatic Adenocarcinomatous deposits. (H\&E, x100). B. Permanent section of the same showing features of Metastatic Adenocarcinomatous deposits. (H\&E, x100). 